\title{
MATHEMATICAL MODELLING OF OPTICAL DENSITY ON THE EXAMPLE OF PRODUCING RASTERIZED FILMS
}

\author{
Bohdan Durnyak ${ }^{1}$, Mikola Lutskiv ${ }^{1}$, Vitalij Buben ${ }^{1}$, Petro Shepita ${ }^{1}$, Georgij Petriaszwili ${ }^{2}$ (D), \\ Zuzanna Żołek-Tryznowska ${ }^{2}$ ii \\ ${ }^{1}$ Ukrainian Academy of Printing, Lviv, Ukraine \\ ${ }^{2}$ Warsaw University of Technology, Institute of Mechanics and Printing, Warsaw, Poland
}

\begin{abstract}
A mathematical model of optical density of rasterized films has been improved, which has significantly increased the calculation accuracy of the optical density, especially at low intensities of the light areas of the image of 0-5\%. The results of the simulation have been presented in the form of graphs of the optical densities characteristics for various parameters and their properties have been analysed.
\end{abstract}

Key words: optical density, rasterized film, simulation model, mathematical modelling.

\section{PREFACE}

Modern production of printing forms is characterized by a high degree of digitization. However, there are examples of using photographic films to perform various tasks. The quality of printing products is determined by the factors that lead to the information changes in reproduction. The factors are associated with the reproduction technological conditions and the nature of the elements that make the information, the manufacture of rasterized printing plates and the printing process in the presence of various effects. Therefore, to improve the quality of the finished products at each stage of the technological chain of the manufacturing process of printed matter, it is necessary to monitor and evaluate certain parameters. Each technological stage is performed on a specific equipment, the less we have the control checks of the parameters, the harder it is to detect various influences, distortions, the waste origin, and it is difficult to optimize certain processes (Baranovskyj et al, 2013; Blanter, 1999; Predko, 2009). One of the most important and complicated process is the process of making films and preparing for direct recording on a plate, changing the size of raster elements in the process of reproduction, increasing (dot gain) of the raster elements area of the image when manufacturing the plates (copying), on the imprint at the contact area of the plate or offset canvas with the paper. The dot gain of the ink layer during the printing process is significant and could be 10-20\% (Nazar et al, 2011; O'Kvin, 2001; Pashulja, 2011; Predko, 2009).

The complexity of the problem is that far not all operations of printing plate manufacturing are controlled. For example, it is now possible only to have a visual supervision of the development of printing plates during their manufacturing. Printing plates are controlled at the end of the process. Instrumentally, the quality of the films is estimated predominantly by a densitometer in the transmitted light at the stage of its withdrawal from the development unit (Nazar et al, 2011; Pashulja, 2011; Predko, 2009). In simple densitometers, there is the problem of the accuracy of determining the optical density of the films in percent, especially in light and dark shades. There is a problem of determining and quantifying the quality of films at the stage of preparation for lighting and recording. Therefore, modelling, calculating and evaluating the quality of raster plates is an urgent task when preparing images for printing.

\section{BACKGRAUNDS}

Modern densitometers are devices for measuring the intensity of reflected or transmitted light, on the basis of which one can determine the optical density, relative area of the printing elements, their geometric dimensions, the optical density of the film or plate in percent, etc. (Blanter, 1999; Pashulja, 2011). Manufacturers of densitometers (the Netherlands, Germany, etc.) for some reason do not provide the algorithms for calculating individual parameters based on the measured intensity of the reflected or transmitted light. Therefore, one should highlight the specific problem of the method for determining the optical density of the film in percent in the transmitted light by separate densitometers, especially in light and dark shades, the measurement error may be tangible (Nazar et al, 2011; Pashulja, 2011; Predko, 2009). 
In the process of manufacturing films and the printing plates, the received imprints, one is constantly dealing with the concept of optical density and other related concepts, whose indicators are defined in Ukrainian standard DSTU 4334-2004, as well as in other sources (Pashulja, 2011). The non-transparent (transparent) areas of the photo films (negative, slide) are estimated by their optical densities measured in the transmitted light. The shade parameters on a raster photo-plate are determined by the relative area of the raster elements in percent of the photo films area, occupied by raster elements on the basis of the measured integral optical density of the image and they are converted into the value of the relative area by the Murray-David formula (Pashulja, 2011). The minimum and maximum optical densities of the photo films are intended for the production of offset plates in accordance with DSTU ISO12647-2 $D_{\min } \leq$ $0,15, a D_{\max } \geq 3,5$. The optical density of transmittance of the raster element should not exceed 0.1 of the density of the photo film background and should not be greater than 0.15 (Nazar et al, 2011; Pashulja, 2011; Predko, 2009).

In most cases, in the printing industry they use digital images that are processed by computer graphics packages, and their quality is evaluated visually, and therefore objectively. The transformation of digital images into a raster photo film and the manufacture of a printing plate is carried out using CtP technology (Kuznetzov, 2002; Lutskiv, 2012; Predko, 2009). The existing methods of digital image processing operate the levels (gradation) of grey that are within [0.255] and operate with arrays of numbers (Gonsales, 2012). Instead, the printing image is much more complex, it has a raster structure, the information carrier is the area of raster elements that have a given shape and linearity (Baranovskyj et al, 2013; Havrysh et al, 2016; Kuznetzov, 2002; O'Kvin, 2001). Therefore, the existing diverse methods for processing digital images (Gonsales, 2012) cannot be directly applied for the analysis and evaluation of raster plates. In the available sources, the light transmittance is determined by the logarithmic expression of the inverse value of the transmittance coefficient for calculating the optical density, instead, based on the measured integral optical density, the images are converted into the values of the relative area according to the Murray-Davis formula which help evaluate the quality of the plates (Kuznetzov, 2002; Pashulja, 2011). However, these formulas are rarely used to calculate, design and analyse the graphic characteristics of raster plates.

The purpose of the work is to improve the existing formulas to determine the gradation characteristics of raster plates, to develop a simulator to calculate and analyse their properties at different effects.

\section{MATEMATICAL MODELLING AND RESEARCHES}

Initially, the term "densitometry" had been used for the notion "measurement of the blackening degree"; it was lately significantly expanded due to various studies of objects and the use of this measuring technique. The degree of blackening is being measured on negatives and positives, black and white, transparent and opaque originals, and on photographic materials and coloured imprints. Objects of measurement can be multi-coloured or raster. Modern densitometers are devices for measuring the intensity of reflected or transmitted light, on the basis of which the optical density, the relative area of printed elements, linear raster and other parameters are determined (Pashulja, 2011). However, not all printers and technologists have enough knowledge concerning the producing qualities of densitometers' analyses, evaluating and controlling the quality of individual technological steps of preprinting and printing processes.

Let one briefly consider the work of the reflecting densitometer, which serves to measure the reflected light, which is most often used by printers to measure on the printed imprints when adjusting the colour apparatus and controlling the imprints quality (Blanter, 1999; Pashulja, 2011). The measurement is based on the difference between incident light on the measuring surface and the amount of reflected light, which depends on the ink layer thickness. Mathematically, we are dealing with the ratio of the incident light and reflected light. Here is a simple example. The amount of incident light on the measured object is taken as 1 . Only the 10th part was reflected, and the rest was absorbed. The next calculation is $1 / 0.10=$ 10. The absorption rate is 10 . However, the densitometer does not indicate the degree of absorption, but only the part of the colour, which is determined and calculated by the decimal logarithm of the absorption rate. The basis of the application of the logarithm is the Weber-Fechner psychophysical law, which states that the visual sense of a person chooses the tone's impression in a logarithmic form (Baranovskyj et al, 2013; Blanter, 1999; Pashulja, 2011). The logarithm calculator is simple and understandable. In number 1 , it is equal to 0 , at $10=1$, at $100=2$, at $1000=3$, and so on.

Non-transparent (transparent) areas of photo-films (negatives, slides) are being evaluated for their optical densities in transmitted light using densitometers. The optical transmittance density in the 
incident light of a photo film is usually determined by the expression (Blanter, 1999; Kuznetzov, 2002; Pashulja, 2011):

$D_{0}=\lg \frac{1}{T}$, or $D_{0}=\lg \frac{F_{0}}{F}$.

where $F_{0}$ - is the flux (intensity) of incident light on the object of measurement, $F$ - is the light flux transmitted to the object of measurement.

T-factor - is the ratio of global fluxes that is transmitted through the measuring sample to the light streams given as a sample. As it has been already mentioned, in simple densitometers, there is the problem of the accuracy of determining the optical density of the photomicrograph at light and dark areas. From the expression (1), it is clear that at low values of light flux $F$ the optical density will increase significantly. For the convenience of calculations, the intensity of light fluxes in percent $\left(F_{0}=100 \%\right)$ is used. To increase the accuracy of calculations, the initial value of $F$ flux is increased by $1 \%$. With such assumptions, the expression (1) for calculating the optical density will look as:

$D_{0}=\lg \left(\frac{100}{F \%}+1\right)$

On the basis of expressions (1) and (2), the optical density of the photo-film in incident light for the small values of intensity has been calculated (Figure 1).

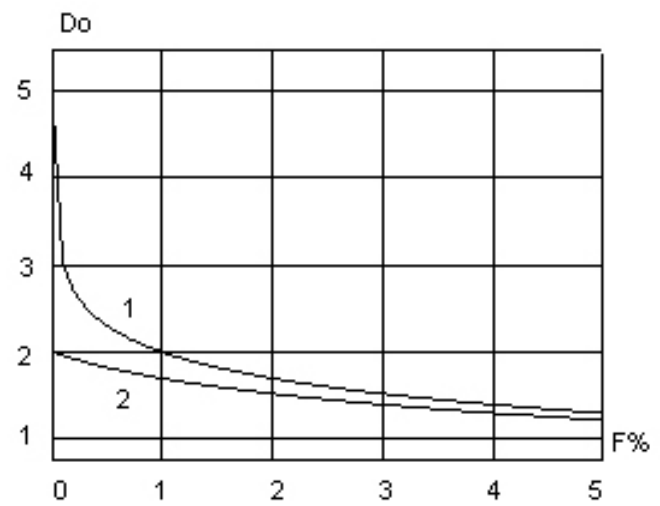

Figure 1: The optical density of the photo-film at the small values of the light intensity 1 - is calculated by the formula (1), 2 - by the expression (2)

The calculated initial value of optical density (1) is more than 30 , which is rounded to 5 for convenience of comparison. At the flux intensity $F=0,5 \%$, the absolute error of optical density is 0,481 , and at $F=1 \%$, the error is 0,21 units. Consequently, the initial flux of the displacement of the intensity of the flux increases the accuracy of the calculation of the optical density at small fluxes. Similarly to expression (2), we present here the formula for the negative:

$D_{0}=\lg \left(\frac{100}{100+F \%}+1\right)$

As an example, the calculated characteristics of the optical density for positive and negative are presented in Figure 2. 


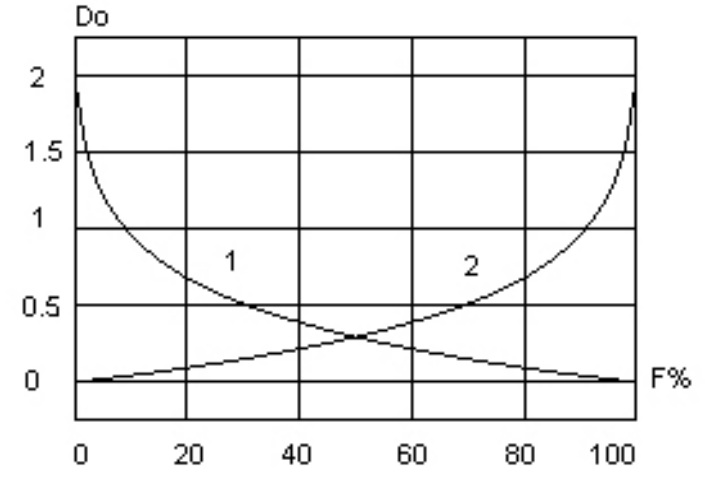

Figure 2: The optical density of the photo-film 1 - positive, 2 - negative

Characteristics of the optical densities of the films for positive and negative are symmetrical, and this confirms the validity of the suggested expressions for the calculation of the optical density. The influence of the change in the intensity of the light flux on the optical density has been studied. The film optical density depends on its properties, for example, the photo-film background and other influences. The effect of decreasing the light flux intensity on the optical density has been researched. The results of calculations are presented in the form of a complex of characteristics of the optical density of the gradual decrease in the flux intensity F0 by 0.9; 0.8; 0.7; 0.6; (Figure 3).

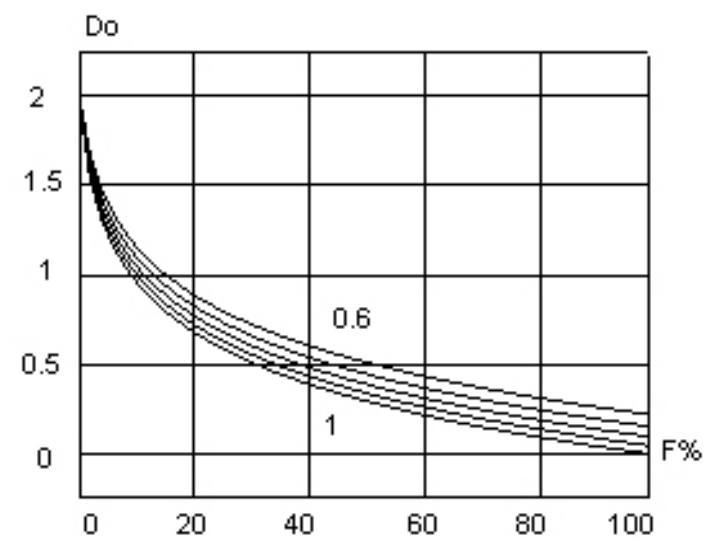

Figure 3: A set of characteristics of photo-plates optical densities at decrease of a light flux intensity

The initial value of the plate optical density does not depend on the intensity of the light flux and is $D_{0}=2$. Nominal intensity of the light flux is F0 $=100 \%$, the characteristic of the optical density is located below, gradually decreases and in the end goes to zero. With a decrease in the light flux intensity, the characteristics are shifted upwards, and the optical density variation from zero is $0.045 ; 0.0968 ; 0.153$; and 0.219 with a tolerance of 0.15 . Consequently, the calculation of the optical density of the photo-film in expression (3) is not very sensitive to the change in the intensity of the light flux, which is its advantage. The analysis of the optical density properties of the plate has been carried out under the condition that the plate substance is homogeneous on the whole surface, and transmittance coefficient changes linearly within $[0 \leq \mathrm{F} \leq$ of $100 \%]$. However, during the stage of image screening the correction is made, due to this the transmittance coefficient, in most cases, is nonlinear. Therefore, the researches of the film optical density at a nonlinear transmittance coefficient are of considerable practical interest. A block diagram has been worked out with the simulator in the package of Simulink, that on the basis of the expression (2) calculates and defines the characteristics of the plate optical density for nonlinear transmittance coefficient (Figure 4): 


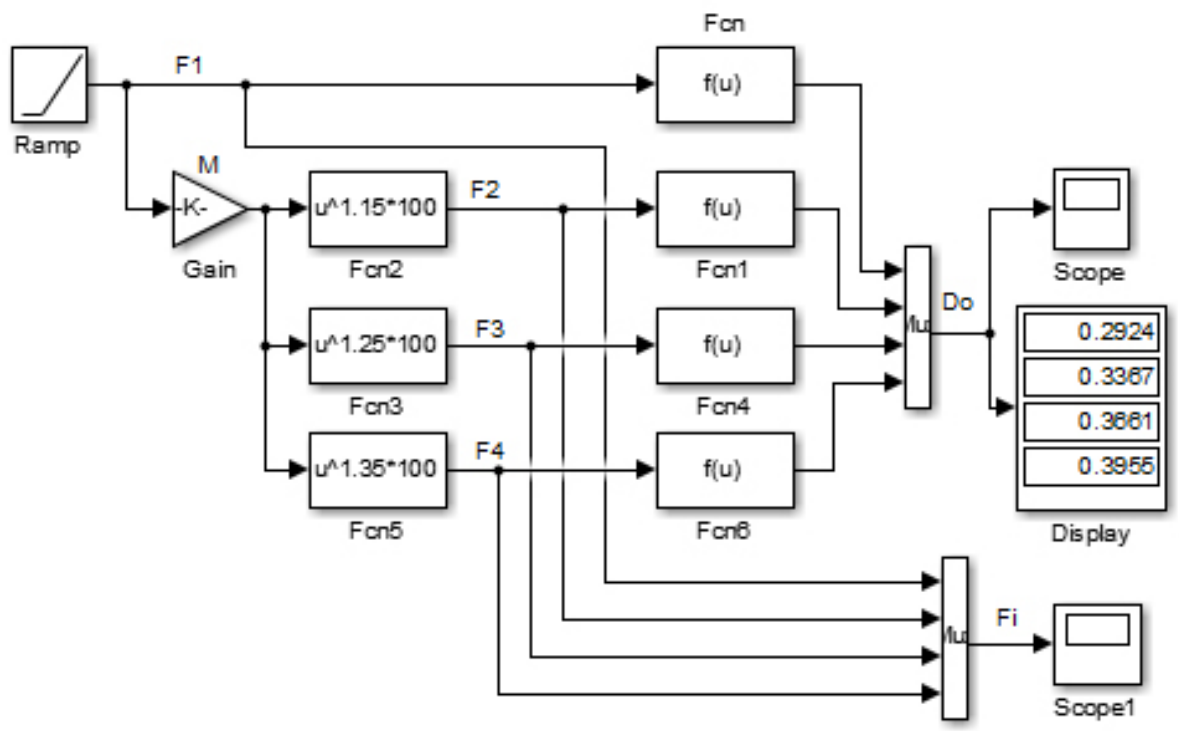

Figure 4: A block diagram of the simulator to define characteristics of the plate optical density for nonlinear transmittance coefficient

The Ramp block generates the linearly increasing Fo light flux, that is scaled by the Gain block. In the first row of math functions blocks Fcn, there are programs that form rated nonlinear coefficients of reduction by the expression $T=U^{v}$, which, after de normalizing, turn into nonlinear light fluxes of $F_{2}, F_{3}, F_{4}$, that are visualized by the Scope 1 block and simultaneously supplied to the output of the second row of math functions blocks Fcn, that calculate films optical density for nonlinear transmittance coefficients, visualized by the Scope block. More sufficient data of separate blocks parameters are shown in Figure4. The results of simulation of nonlinear light fluxes are shown in Figure 5:

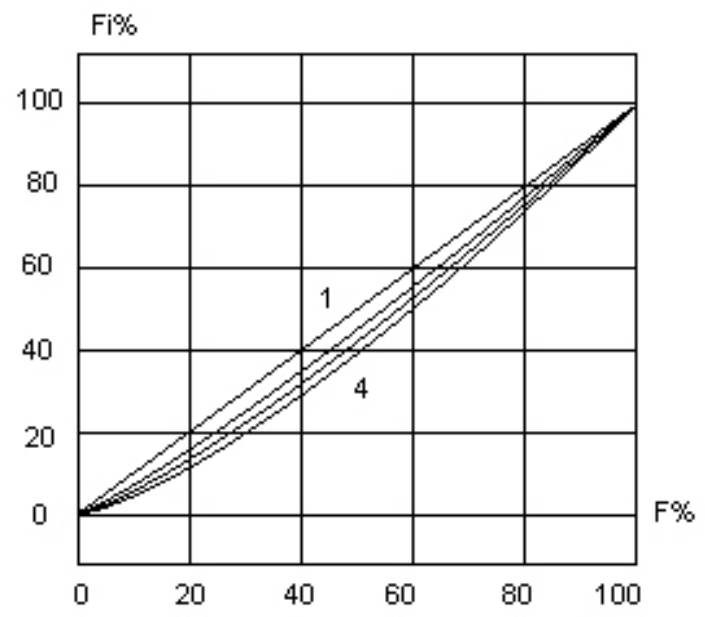

Figure 5: Parameters of nonlinear light fluxes

Upper parameter is linear. Nonlinear parameters of fluxes are placed lower in the form of the curved graphics. Maximal parameters deviations from the linear is on the middle fluxes and constitute 5.2; 8,2; $11,0 \%$, that is quite small in general. Physically non-linearity of the transmittance coefficients causes the distortion of raster elements sizes (expending) during the process of reproduction of the photo-film contamination production, as well as copying from a photo-film or direct record on the film. The results of simulation of nonlinear transmittance coefficient influence on the film optical density are shown in Figure 6 in the form of the set of parameters: 


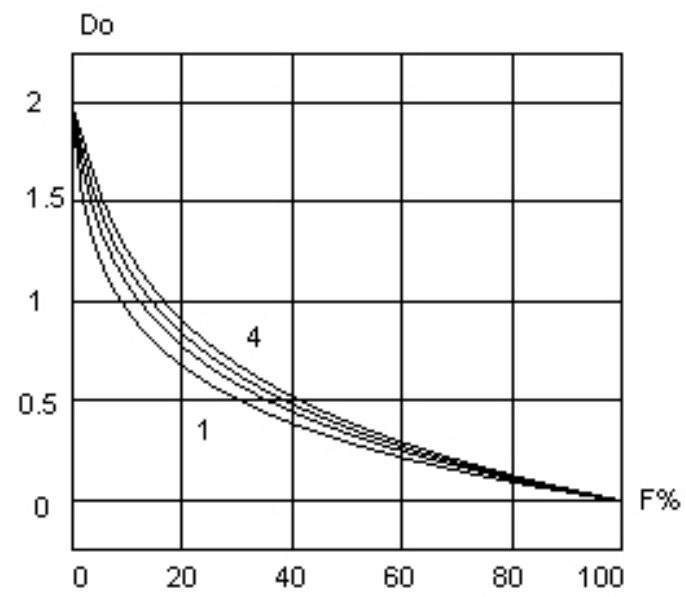

Figure 6: A set of parameters of the plate optical densities for nonlinear transmittance coefficients

Slight deviation of light fluxes from linearity $(5,2 ; 8,20 ; 11,0 \%)$ considerably shifts the parameters of the film optical densities to the right. It is offered to assess the influence of non-linearity of transmittance coefficient on the optical density by the maximal deviation in the parameter from the first one $\left(E=D_{i}-D_{0}\right)$. The results of simulation of the deviation of the film optical density from the nominal one for different light fluxes are shown in Figure 7:

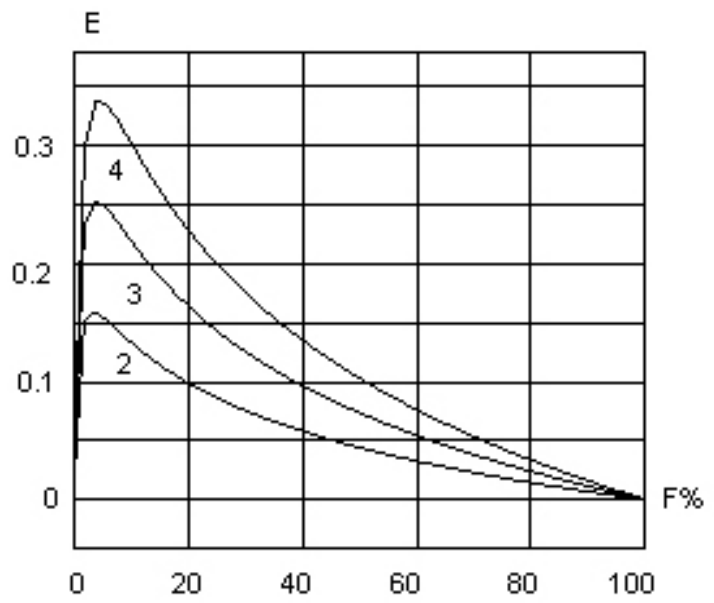

Figure 7: Deviation of the photo-film optical density from the nominal one for light fluxes variation

Slight deviation of light fluxes from the linearity $(5,2 ; 8,20 ; 11,0 \%)$ causes large deviations of the optical density on small fluxes ( $F=5-100 \%$ ). Their maximal values are 0,$34 ; 0,25$ and 0,16 units at tolerance of not more than 0,15 units. Thus, the film optical density is most sensible to expanding on light tones. The increase of plate optical density at non-linearity of transmittance coefficient must be taken into account as distortions of raster elements during the process of reproduction, the so-called expanding, i.e. an increase of area (sizes) during the production of rasterized films, as well as copying from a photo-film or direct record on a plate in the CtP systems, though it is often ignored. To control the expansion of a photo-film, it is possible to use a densitometer by measuring the density of the controlled scale instead of the area.

\section{CONCLUSIONS}

A mathematical model of optical density of rasterized films has been improved, which has significantly increased the calculation accuracy of the optical density, especially at low intensities of the light flux of 0$5 \%$. The results of the simulation have been presented in the form of graphs of the optical densities characteristics for various parameters and their properties have been analysed. 
The block diagram of the simulator Simulink in the Matlab package has been worked out, which provides an opportunity to calculate and design a family of the optical densities characteristics for various parameters, analyses, and practical application. The results of the simulation of the linear reduction influence of the light flux intensity in the form of a set of the optical densities characteristics of a photofilm have been presented and it has been found out that even double reduction of a flux has little effect on the optical density, the finite deviation of the optical density is 0,219 at tolerance of 0,15 units. Thus, plate optical density is quite insensitive to the linear changes of the flux intensity.

The influence of the nonlinearity of the transmittance coefficient has been studied, caused by distortions of the geometric dimensions of raster elements (expanding) in the reproduction process of the plate contamination production, as well as copying from a photo-film or direct record on a plate. It has been found out that slight deviation of the transmittance coefficient, with its maximal values of 5,$2 ; 8,2 ; 11,0 \%$, causes significant deviation of the optical density on small fluxes ( $F=5-10 \%$ ), their maximum values are $0.34,0.25$ and 0.16 units at tolerance of not more than 0.15 units, so the expanding (the increase of the area of the raster elements) must be taken into account while raster plate production, which is often ignored. To control the expansion of a photo-film, it is possible to use a densitometer by measuring the density of the controlled scale instead of the area. The obtained results of the research can be used for preparing images for screening, as well as for photo-films production and control.

\section{REFERENCES}

[1] Baranovskyj V., Lutskiv M., Fil L., Chernozubova G.: "Construction and analysis of screening characteristics", Journal Naukovi zapysky UAP 4, 131-138, 2013.

[2] Blanter D.: "Scanning and screening images", (ECOM, Moscow, 1999.)

[3] Gonsales R., Vuds R.: "Digital image processing", (Tekhnosfera, Moscow, 2012.)

[4] Havrysh B., Durniak B., Tymchenko O., Yushchyk O.: "Vidtvorennia zobrazhen rastrovymy skanuiuchymy prystroiamy", (UAD, Lviv, 2016.)

[5] Kuznetzov J.: "Technology for image information processing", (Petersburg Institute of Printing, St. Petersburg, 2002.)

[6] Lutskiv M., Buben V.: "Construction of the characteristics of demodulation linear tint chart", Journal Computer printing technology 2 (40), 109-115, 2018.

[7] Lutskiv M.: "Digital printing technology", (Ukrainian Academy of Printing, Lviv, 2012.)

[8] Nazar I., Lazarenko E., Jakutsevych S.: "Offset printing options: management and influence factors", (UAP, Lviv, 2011.)

[9] O’Kvin D.: "Dopechatnaja podgotovka. Rukovodstvo dizajnera", (Vil'jams, Moscow, 2001.)

[10] Pashulja P.: "Standardization, metrology, compliance, printing quality", (UAP, Lviv, 2011.)

[11] Predko L.: "Prepress Process Design: tutorial", (UAP, Lviv, 2009.)

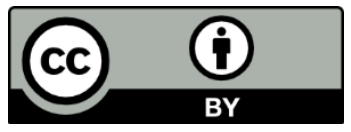

(C) 2020 Authors. Published by the University of Novi Sad, Faculty of Technical Sciences, Department of Graphic Engineering and Design. This article is an open access article distributed under the terms and conditions of the Creative Commons Attribution license 3.0 Serbia (http://creativecommons.org/licenses/by/3.0/rs/). 